\title{
The Study of the Dangerous Goods Transport Subsidies in the Traffic Network
}

\author{
Lingfei zhu \\ School of Traffic and Transportation, \\ Beijing Jiaotong University, No.3 Shangyuancun, Haidian district, \\ Beijing, China \\ Email: 14120766@bjtu.edu.cn
}

\begin{abstract}
In the transportation network has a special transportation problem, is the problem of transport of dangerous goods, we all know that transport of dangerous goods in the event of accident, caused the harm is far greater than ordinary traffic accident, so the study of the transport is crucial. In this paper, considering the risk equilibrium, turning it into a bi-level programming problem, and then use particle swarm optimization algorithm to work out, it is concluded that increasing government subsidies can reduce the total risk and the time cost and increase network network requirements under the condition of the conclusion of present a tendency of increasing time cost. These results provide theory basis for dangerous goods transportation decisions for the government, so as to improve the safety of the transport of dangerous goods. Limited to the length and capacity, the number of dangerous goods vehicles ratio and the influence of social factors such as vehicles in the morning and evening peak level remains to be further research.
\end{abstract}

Keyword-risk equilibrium;government subsidies; dangerous goods; time cost; Traffic demand

\section{INTRODUCTION}

In the study of dangerous goods transportation network fees issue, The government is the management department, the object is the dangerous goods transportation vehicles in the network, the influence factor is the social vehicle. Transport of dangerous goods usually very important, once the accident occurs, it may cause the larger damage than normal accident. Before, many scholars have done research in this regard, and proposed some methods. Bianco considered the possibility of an accident, dangerous goods transport of dangerous goods vehicle traffic accident occurred in a greater probability of major sections of the restrictions, and proposed probability limit method【1】. Akgun considers the transportation risk of the optimal path, and directly seeks the alternative route of the optimal path for the transportation cost of dangerous goods, finally present the path substitution method【2】.Zografos, the maximum risk of road section is considered as the constraint conditions, and the maximum risk of each section is limited, and put forward the risk limit method.

In view of the theoretical and practical features of the above mentioned methods, this paper will adopt the expected risk limit of road section as an optimization objective to realize the equilibrium of network risk【35】.

\section{CONSTRAINT CHARACTERISTIC OF THE MODEL}

During the transportation of dangerous goods, the decision-making includes two aspects: One is the government administration department, and the other is the basic vehicle unit in the network (including dangerous goods vehicles and ordinary social vehicles). As a result, the transportation network optimization problem can be described as a bi-level programming problem. Government management department for the top decision makers, decision making includes two aspects: one is on distribution of dangerous goods vehicles in the network path, second is in the network on the sections of the road vehicle of subsidies to the society. Vehicles in the network society is for decision makers, the top decision makers can not directly control network in ordinary social vehicle routing, but merely through the impact it could have its path selection【6】.

\section{RESEARCH METHODS}

The optimization objective of the upper level programming is the minimization of the risk limit for the section of the road section. Decision makers is the government management departments, subsidies for the total government is budget constraints, decision-making including distribution of dangerous goods vehicles path and execute road vehicles subsidies to the society.

$$
\min R
$$

(1)

$$
x_{i j 2} \cdot\left(\rho_{i j}+\varepsilon x_{i j 1}\right) \leq R
$$

(2)

$$
\sum_{i j} x_{i j 1} \cdot \gamma_{i j} \leq B
$$

Formula 1 is the target function for the upper level,it means minimizing the risk limit for sections of a section. ${ }^{\mathrm{X}_{i j 1}}$ means Social vehicle number $x_{i j 2}$ means the number of dangerous goods vehicles on the road. $\rho_{i j}$ is the population of the road section $\mathrm{ij}, \mathrm{R}$ is 
expressed as the upper risk limit for sections, ${ }{ }^{i j}$ is the subsidies for social vehicles for sections of the road $\mathrm{ij}, \mathrm{B}$ is the total subsidy budget of government 【7】.

The lower level is a mixed traffic equilibrium assignment problem. Lower social vehicles in the upper subsidies choose the path and eventually reach equilibrium on the basis of decision-making in the freedom. Get road traffic and react on the upper target, the road section traffic costs determined by the traffic of all vehicles. Considering the generalized cost, the cost of the two types of vehicles has different functions, each kind of vehicles flow on itself and also influence another cost function of the vehicle. Variational inequality is used to determine the mixed equilibrium section flow form $X_{i j}^{*}$,then the mixed equilibrium under fixed demand: the social vehicle routing traffic and road traffic and (7) is the non negative constraint of traffic flow and path flow in social vehicles.

$$
\sum_{m} f_{2 m}^{r s}=q_{2}^{r s}
$$

(8)

$$
x_{i j 2}=\sum_{r, s} \sum_{m} f_{2 m}^{r s} \delta_{i j, m}^{r s} \quad \forall r, s, i, j
$$

$$
x_{i j 2} \geq 0, f_{2 m}^{r s} \geq 0
$$

(8) is the demand constraints for dangerous goods vehicles,(9) is the relationship between the dangerous goods vehicle routing traffic and road traffic, (10) is the non negative constraints for traffic flow and path flow of dangerous goods vehicles.

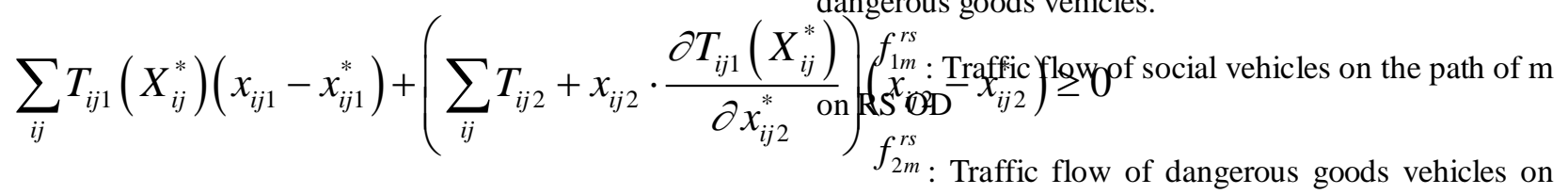

$$
T_{i j 1}\left(X_{i j}\right) \text { is the social vehicle section cost }
$$

function, so we define it

$$
\begin{aligned}
& T_{\mathrm{ij} 1}\left(X_{\mathrm{ij}}\right)=t_{i j}\left(x_{i j 1}, x_{i j 2}\right)+\theta * x_{i j 2}-r_{i j}=t_{i j}^{0}\left\{1+a\left[\left(x_{i j 1}\right.\right.\right. \\
& T_{i j 2}\left(X_{i j}\right) \text { is dangerous goods vehicle section cost }
\end{aligned}
$$
function:

$T_{i j 2}\left(X_{i j}\right)=t_{i j}\left(x_{i j 1}, x_{i j 2}\right)=t_{i j}^{0}\left\{1+\alpha\left[\left(x_{i j 1}+x_{i j 2}\right) / C_{i j}\right]^{\beta}\right\}$

$t_{i j}(x)$ represents the time impedance function of the traffic flow as the independent variable in the road

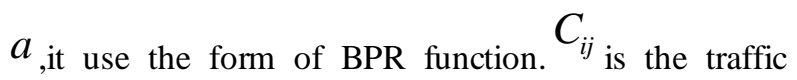
capacity of the road $i j, \theta_{\text {is the risk cost perception }}$ coefficient of social vehicles for dangerous goods vehicles.

\section{RESTRICTION CONDITION:}

The travel behavior of the social vehicles only considers the minimum cost, and the travel behavior satisfies the user's optimal principle.

(5)

$$
\sum_{m} f_{1 m}^{r s}=q_{1}^{r s}
$$

$$
x_{i j 1}=\sum_{r, s} \sum_{m} f_{1 m}^{r s} \delta_{i j, m}^{r s} \quad \forall r, s, i, j
$$

(6)

$$
x_{i j 1} \geq 0, f_{1 m}^{r s} \geq 0
$$

Equations (5) is the vehicle demand constraints for the society, equations (6) is the relationship between the path of $\mathrm{m}$ on RS by OD

$q_{1}^{r s}$ : OD fixed number of social vehicles and fixed demand for RS $\left.\left.\left.x_{i j 2}\right) / G_{2}^{s j}\right]^{\beta}\right\}+\theta * x_{i j 2}-r_{i j}$ fixed for the fixed

$x_{i j 1}$ : Social vehicle road traffic, the road on the ij social vehicle number $\varepsilon$ : Sections on the vehicle conversion coefficients for exposure of the population, society take 2 said the standard car unit under the condition of social division of the vehicle by personnel number is 2 [9].

\section{CASE STUdy}

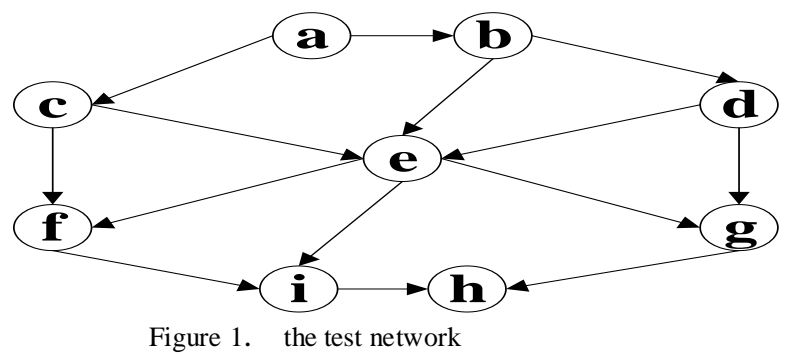

As shown in Figure 1, there is a OD pair (a to $h$ ) have 9 sections and 14 nodes, $\mathrm{a}$ and $\mathrm{h}$ as the starting point of the network. Section latency function uses BPR function, Social vehicle conversion factor $\varepsilon$ take 2,exposure to the population of units of (a hundred),the free flow time $t_{i j}^{0}(\mathrm{~s})$ and the traffic capacity of the section such as $C_{i j}$ are shown in table 1: 
TABLE I.

PARAMETERS OF THE TEST NETWORK

\begin{tabular}{ccccccccccccccc}
\hline road & $\boldsymbol{a}-\boldsymbol{b}$ & $\boldsymbol{a}-\boldsymbol{c}$ & $\boldsymbol{b}-\boldsymbol{d}$ & $\boldsymbol{b}-\boldsymbol{e}$ & $\boldsymbol{c}-\boldsymbol{e}$ & $\boldsymbol{c}-\boldsymbol{f}$ & $\boldsymbol{d}-\boldsymbol{e}$ & $\boldsymbol{d}-\boldsymbol{g}$ & $\boldsymbol{e}-\boldsymbol{f}$ & $\boldsymbol{e}-\boldsymbol{g}$ & $\boldsymbol{e}-\boldsymbol{i}$ & $\boldsymbol{f}-\boldsymbol{i}$ & $\boldsymbol{g}-\boldsymbol{h}$ & $\boldsymbol{i}-\boldsymbol{h}$ \\
\hline$t_{i j}^{0}$ & 4 & 3.5 & 5 & 6.8 & 5.6 & 6.8 & 7 & 3.8 & 3.6 & 4 & 3.8 & 4 & 7 & 5.4 \\
$\rho_{i j}$ & 3.0 & 2.1 & 1.9 & 8.2 & 8.1 & 7.9 & 7.5 & 5.4 & 2.2 & 3.1 & 2.5 & 2.6 & 6.8 & 7.3 \\
$C_{i j}$ & 25 & 31 & 29 & 25 & 18 & 16 & 21 & 25 & 24 & 14 & 16 & 15 & 22 & 18 \\
\hline
\end{tabular}

A. The impact of the total government subsidy budget

Keep the total network traffic demand is 200, dangerous goods vehicles in a proportion of the total traffic demand of 5\%,risk cost perception coefficient a was 1 ,adjust the total government budget increase from 4000 to 8000,get results as shown in Figure 2

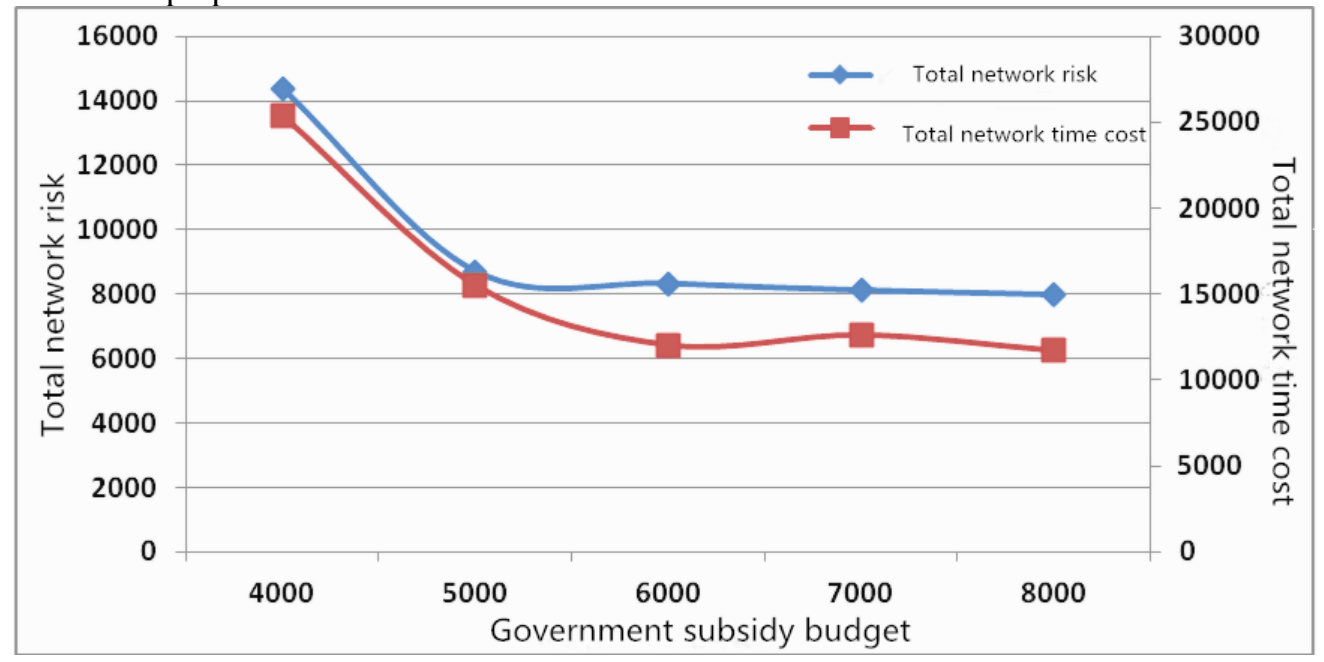

Figure 2. the influence of total governmental subsidy budget

Through the results, it can be seen that when the government subsidies total budget increases from 4000 to 8000 in the process, no matter the network to the total risk and the total time costs are significantly lower, the dangerous goods transport network from two aspects of risk and traffic conditions continue to improve. This suggests that the risk equilibrium as the optimization goal can also reduce the risk of total network, network congestion and improve the transportation condition. Figure curve in the rate of descent has intuitive sense, subsidy budget increased from 4000 to 5000, during the process of network has dramatically improved total risk and the total time cost, but with the further increase of the budget, subsidies reduce the risk of network and improve the network congestion effect started to not obvious, this has certain practical reference value for government management departments. Government regulators can fully save cost of subsidies to achieve control effect, reduce the economic costs of waste.

\section{B. Effects of total network traffic demand}

Keep dangerous goods vehicles in a proportion of the total traffic demand is $5 \%$, cost risk perception factor $\theta$ is 1 , total government subsidies budget for 6000 , adjust the total network traffic demand increase from 100 to 100, the results are shown in Figure 3.

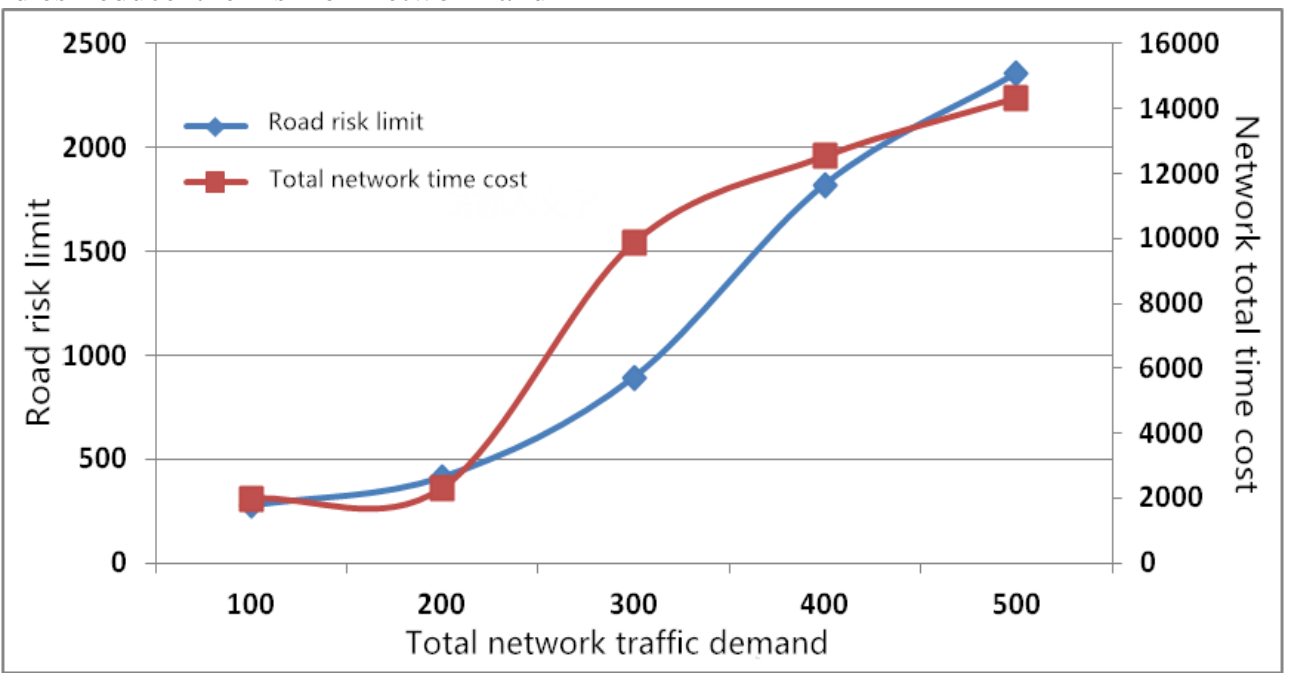

Figure 3. Influence of Total Traffic Volume in the Network 
According to the results shown in Figure 3, we can see the impact of the risk cap and the total time of the network, the impact of the changes in the cost of the total time, the two are a sustained upward trend. The increase of the risk limit of the road section is because the number of dangerous goods vehicles is increasing, and the risk value of the road section is also increased. When the traffic demand is small, the congestion effect is not obvious; the total time of the network has little change. With the increase of the number of vehicles, the impedance of the network is increasing rapidly, and the congestion degree is also increasing. With the increase of traffic demand, the network is congested and the total time of the network is also gradually slow.

\section{CONCLUSION}

For dangerous goods transportation network, this article studies the network dangerous goods vehicles and social vehicles mixed, takes the risk balance as the optimization goal, considers social vehicle risk sexy perceived cost; according to the system optimum principle of dangerous goods vehicles for path allocation; at the precalculation based on network social vehicles subsidies, through the influence of the traffic cost influence the path selection; ultimately makes under the mixed traffic equilibrium, equilibrium of network risk obtained represent the greatest. The conclusions of the specific results are as follows:

The total government subsidy budget for network total risk and the influence of the total time cost are analyzed. The Government is subsidized by reasonably setting the total budget management, on the basis of the economic cost saving significantly reduced risk of total network, vehicle total time cost in the network is also a significant improvement[10].

The total time in total traffic demand for network cost and the impact of road risk limit are analyzed. Of vehicle number in network increasing road impedance increases, can lead to network congestion increases, the number of dangerous goods vehicles increases also led to the network of road risk limit increased.

In the research of the defects, the setting of the government budget is relatively simple and cannot ensure the actual situation, and can further study and discuss with the actual combination. In addition, the paper considers the government centralized management of dangerous goods vehicles, and the corresponding practical operation is more complex, as yet to be further studied.

\section{REFERENCES}

[1] Bianco L., Caramia M., Giordani S. A bilevel flow model for hazmat transportation network design $[\mathrm{J}]$. Transportation Research Part C, 2009, 17: 175-196.

[2] Akgun V., Erkut E., Batta R. On finding dissimilar paths [J] European Journal of Operational Research, 2000, 121(2): 232 246.

[3] Zografos K.G., Androutsopoulos K. N. A decision support system for integrated hazardous materials routing and emergency response decisions $[\mathrm{J}]$. Transportation Research Part C: Emerging Technologies, 2008, 16(6): 684-703.

[4] Wardrop J. G. Some theoretical aspects of road research [J] Proceedings of the Institute of Civil Engineers, 1952.

[5] Jiang Yang. Research on traffic network design based on structure and random properties[D]beijing: Beijing Jiaotong University ,2011

[6] Brotcorne L., Labbe M., Marcotte P., Savard G. Joint design and pricing on a network [J]. Oper.Res, 2008, 56(5): 1104-1115.

[7] Marcotte P., Mercier A., Savard G., Verter V. Toll Policies for Mitigating Hazardous Materials Transport Risk [J]. Transportation Science, 43(2): 228-243.

[8] Beckman M. J., McGuire C. B., Winsten C. B. Studies in the Economics of Transportation [M]. Yale University Press, NewHaven, C.T., 1956.

[9] Lina Zhang. Road transport of dangerous goods network design model and algorithm[D]lanzhou: Lanzhou Jiaotong University, 2013

[10] Shanshan Peng. Considering fairness constraint of dangerous goods transportation network design research[D]beijing: Beijing Jiaotong University ,2013 